\title{
Appréciation de l'hydrogénation des acides gras alimentaires dans le rumen de la vache : étude méthodologique préliminaire
}

\author{
M. Doreau 1 , V. Batisse ${ }^{1}$ et D. Bauchart 2 \\ avec la collaboration de J. Flechet ${ }^{1}$ et M. Martinaud ${ }^{2}$
}

\begin{abstract}
${ }^{1}$ INRA, laboratoire de la lactation, Centre de recherches de Clermont-Ferrand, Theix, 63122 Ceyrat;
2 INRA, laboratoire d'étude du métabolisme énergétique. Centre de recherches de ClermontFerrand, Theix, 63122 Ceyrat, France
\end{abstract}

(recu le 7 décembre 1988, accepté le 26 mai 1989)

Résumé - L'hydrogénation des acides gras alimentaires dans le rumen a été étudiée en distribuant à 2 vaches recevant de l'ensilage de maîs 2 sources lipidiques (huile de colza et savons de calcium) en un temps très court (environ $850 \mathrm{~g}$ en $15 \mathrm{~min}$ ), puis en étudiant l'évolution postprandiale de la composition centésimale des acides gras totaux présents dans le contenu duodénal. Dans les 2 cas, les acides linoléque et linolénique paraissent très fortement hydrogénés puisqu'ils n'ont représenté respectivement que 2 à $3 \%$ et 0,5 a $1 \%$ des acides gras à 18 atomes de carbone du contenu duodénal. Les acides gras octadécénoïques ont constitué de 40 à $50 \%$ des acides gras à 18 atomes de carbone : ce pourcentage, peu moditié par l'ingestion de lipides, est lègèrement plus élevé avec I'huile de colza. La proportion d'acide propionique dans le mélange des AGV du rumen a semblé corrélée et a également été plus élevée avec l'huile de colza, ce qui pourrait traduire une modification plus importante des fermentations ruminales.

digestion — vache - acides gras — hydrogénation

Summary - Evaluation of ruminal hydrogenation of fatty acids in cows : preliminary methodological results. Ruminal hydrogenation of fatty acids was studied by an in vivo method. Two lipid sources (rapeseed oil or calcium palm oil soaps) were given to dairy cows very rapidly (about $850 \mathrm{~g}$ within $15 \mathrm{~min}$ ). Duodenal fatty acid pattern was analyzed all day long. With the two diets, linoleic and linoleic acid appeared extensively hydrogenated : they accounted for $2-3$ and $1-2 \%$, respectively of the 18 carbon-fatty acids of the duodenal contents. Octadecenoic fatty acids represented 40 to $50 \%$ of the 18 carbon-fatty acids. This percentage was slightly higher with rapeseed oil than with calcium soaps. Prandial variations were low. The proportion of propionic acid in rumen VFA seemed to be related to this percentage and was higher with rapeseed oil, which could be related to larger variations in ruminal fermentations than with calcium soaps.

digestion - cow - fatty acids - hydrogenation 


\section{INTRODUCTION}

Les matières grasses ingérées par les ruminants subissent dans le rumen une hydrolyse rapide et le plus souvent quasi totale, suivie d'une hydrogénation importante des acides gras polyinsaturés de type C 18:2 n-6, et surtout 18:3n-3. Ces acides gras, particulièrement ceux de la famille des $n-3$, entraînent fréquemment des perturbations de la digestion ruminale des glucides (revue in Bauchart et al., 1985). Ces constatations ont conduit à préconiser l'emploi de matières grasses soit saturées ou mono-insaturées, soit "protégées" de l'hydrogénation dans le rumen. Une diminution de l'hydrogénation pourrait avoir comme conséquences, outre la réduction des éventuels effets négatifs des lipides sur le fonctionnement du rumen, une modification de la composition des acides gras absorbés et donc des acides gras du lait. Aussi est-il important de pouvoir juger de la capacité d'une source lipidique à être hydrogénée dans le rumen.

Les mesures précises d'appréciation de l'hydrogénation des lipides dans le rumen, qui nécessitent la détermination du flux duodénal, sont longues et coûteuses. Pour caractériser une source de lipides alimentaires, il serait nécessaire de disposer d'une méthode d'appréciation rapide de l'hydrogénation. Différentes tentatives ont été réalisées en incubant des matières grasses in vitro. Les auteurs qui ont pratiqué ces techniques ont abouti à un constat d'échec des méthodes utilisées (Brumby $\&$ Storry, 1976). Les résultats obtenus in vitro estiment souvent mal la «protection" contre l'hydrogénation par rapport aux mesures in vivo, parce qu'ils ne tiennent pas compte de la mastication, qui contribue à rompre les structures cellulaires, et parce qu'ils n'intègrent pas le temps de séjour des aliments dans le rumen. Aussi avons-nous décidé d'étudier une méthode d'évaluation in vivo du métabolisme ruminal des acides gras, comparable dans son principe à celle qu'a utilisé Steele (1983), et qui consiste à analyser la composition des acides gras du liquide ruminal ou du contenu duodénal. Nous avons choisi cette dernière solution, bien qu'elle semble moins facilement utilisable pour les mesures de routine, parce que la mesure au niveau duodénal constitue un bilan du fonctionnement du rumen et caractérise les acides gras absorbables, mais aussi parce qu'un prélèvement de liquide ruminal ne prend pas en compte les lipides associés aux particules ou aux bactéries fixées aux particules. Ces dernières, qui représentent une forte proportion des bactéries totales, ont une teneur et une composition en acides gras différents de celles des bactéries libres (Legay-Carmier \& Bauchart, 1989; Bauchart et al., 1989).

Le présent essai constitue la première étape de la mise au point de la méthode. II utilise une technique de surcharge importante en lipides, de sorte que la source de matières grasses étudiée représente près de $80 \%$ de l'apport totale d'acides gras. II consiste à décrire une cinétique de l'hydrogénation, de manière à choisir le ou les points horaires de prélèvements pour des études ultérieures. Enfin, la cinétique duodénale des acides gras longs est complétée par une cinétique ruminale des acides gras volatils, afin d'analyser les relations entre ces 2 profils.

\section{MATÉRIEL ET MÉTHODES}

\section{Animaux et régimes}

Deux vaches laitières taries de race Holstein, d'un poids voisin de $600 \mathrm{~kg}$ et munies de 
canules du rumen et du duodénum proximal, ont été utilisées. Sur une période de 10 jours, ces vaches ont reçu chaque jour $7 \mathrm{~kg}$ de matière sèche d'ensilage de maïs et $850 \mathrm{~g}$ d'acides gras provenant, pour une vache, d'huile de colza, et pour l'autre vache, de savons de calcium obtenus à partir d'acides gras d'huile de palme. Ces régimes ont été respectivement nommées $\mathrm{H}$ et S. L'ensilage a été distribué en un repas à $8 \mathrm{~h}$ 30 et la source lipidique a été introduite par la canule du rumen en 4 parts égales, à $9 \mathrm{~h}, 9 \mathrm{~h}$ 05,9 h 10 et 9 h 15.

La teneur et la composition en lipides des aliments figurent sur le Tableau I.

\section{Prelèvements et analyses}

Des prélèvements de contenus ruminal et duodénal ont été réalisés le dixième jour de la période, à $8 \mathrm{~h} 30$ (avant la distribution d'ensilage), $9 \mathrm{~h}$ (avant l'introduction des matières grasses), $10 \mathrm{~h}, 11 \mathrm{~h}, 12 \mathrm{~h}, 13 \mathrm{~h}, 15 \mathrm{~h}, 17 \mathrm{~h}, 21 \mathrm{~h} 30$ et $1 \mathrm{~h}$ 30. Environ $50 \mathrm{ml}$ de liquide ruminal ont été aspirés à l'aide d'une sonde. Au niveau duodénal, $250 \mathrm{ml}$ ont été obtenus par gravité.

Sur les échantillons de liquide du rumen, la valeur du $\mathrm{pH}$ a été mesurée et la teneur et la composition des AGV ont été analysées par chromatographie en phase gazeuse (Jouany, 1982). Les lipides duodénaux ont été extraits en double, selon une technique dérivée de celle de
Folch et al. (1957), et analysés par chromatographie en phase gazeuse sur colonne capillaire (Bauchart et al. 1987). Les acides gras obtenus ont une longueur de chaine comprise entre 14 et 24 atomes de carbone. Dans l'exploitation des résultats, nous avons considéré uniquement les proportions relatives des acides gras à 18 atomes de carbone. Les différents isomères de position ou géométriques octadécénoïques séparés par la chromatographie ont été additionnés. Parmi les acides gras polyinsaturés, seuls les acides linoléique (C 18:2 $n-6$ cis cis) et linolénique (C 18:3 n-3 cis cis cis), présents dans les aliments, ont été étudiés.

\section{RÉSULTATS ET DISCUSSION}

\section{pH et AGV du liquide du rumen}

L'évolution du $\mathrm{pH}$ avec le régime $\mathrm{S}$ est comparable à celle que l'on observe généralement avec des régimes à base d'ensilage de maïs (Fig. 1). La concentration en AGV suit une évolution inverse. En revanche, le $\mathrm{pH}$ mesuré avec le régime $\mathrm{H}$ est très stable au cours de la journée. II est

Tableau I. Teneur et pourcentage des principaux acides gras des éléments de la ration.

\begin{tabular}{lccc}
\hline & $\begin{array}{c}\text { Ensilage de } \\
\text { maïs }\end{array}$ & $\begin{array}{c}\text { Huile de } \\
\text { colza }\end{array}$ & $\begin{array}{c}\text { Savons de } \\
\text { calcium }\end{array}$ \\
\hline Teneur en acides gras (\% MS) & 2,5 & 98,0 & 83,6 \\
Composition des acides gras & & & \\
(g/100 g d'acides gras) & & & \\
C14:0 & 0,7 & 5,8 & 1,7 \\
C16:0 & 15,4 & 1,9 & 44,5 \\
C18:0 & 2,0 & 61,5 & 3,6 \\
C18:1 & 24,4 & 21,6 & 41,8 \\
C18:2 & 43,9 & 8,2 & 8,1 \\
C18:3 & 8,0 & & 0,3 \\
\hline
\end{tabular}




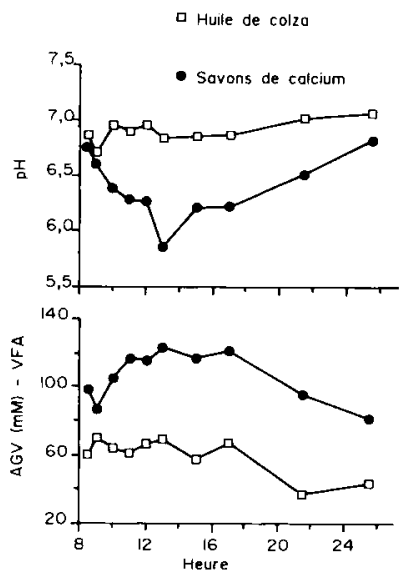

Fig. 1. $\mathrm{pH}$ et concentration en AGV du liquide du rumen.

possible que la cause en soit la faible concentration en AGV, qui pourrait être une conséquence de la réduction des fermentations ruminales souvent observée avec ce type de régime (Doreau et al., résultats non publiés). Inversement, la concentration élevée en AGV pour le régime $S$ traduirait une action modérée des savons sur les fermentations des glucides, confirmant les données de Jenkins \& Palmquist (1984).

L'accroissement de la proportion de propionate dans le mélange des AGV est beaucoup plus sensible pour le régime $H$ que pour le régime $S$, indiquant une modification marquée des orientations fermentaires. Les variations des proportions de butyrate au cours de la journée sont difficilement interprétables, car leur évolution au cours de la journée n'a pas été cyclique (Fig. 2).

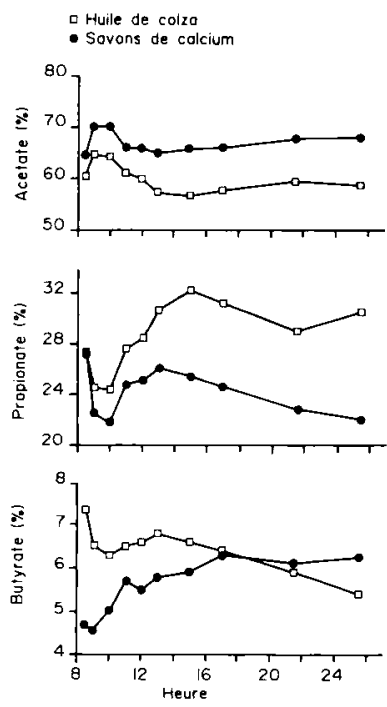

Fig. 2. Proportion des AGV du liquide ruminal (en pourcentage molaire des AGV totaux).

\section{Acides gras duodénaux}

Les proportions des acides linoléique et linolénique ont été très faibles à toutes les heures de la journée (Fig. 3), ce qui paraît indiquer une hydrogénation très élevée de ces acides, présents dans les aliments ingérés en quantités plus importantes pour le régime $\mathrm{H}$ (respectivement 28 et $9 \%$ des acides gras à 18 carbones) que pour le régime $S$ (respectivement 25 et $2 \%$ des acides gras à 18 carbones). Une appréciation de l'hydrogénation peut être obtenue en faisant l'hypothèse d'un flux duodénal d'acides gras égal à la quantité d'acides gras ingérée, ce qui ne constitue qu'une approximation, car ce flux est probablement un peu plus faible (revue in Doreau 


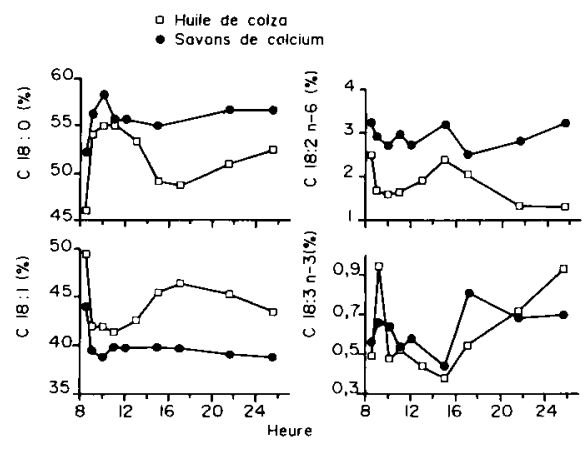

Fig. 3. Proportion des acides gras en $\mathrm{C} 18$ du contenu duodénal (en pourcentage des $\mathrm{C} 18$ totaux).

et al., 1989). L'hydrogénation de l'acide linoléique apparaît alors très élevée; sa contribution au flux duodénal est peut-être liée à son incorporation par les bactéries du rumen, et donc à sa protection contre l'hydrogénation (Bauchart et al., 1989). L'acide linolénique est très fortement hydrogéné avec le régime $\mathrm{H}$, mais probablement plus faiblement avec le régime $S$, pour lequel il provient essentiellement de l'ensilage. Ce résultat est surprenant car l'hydrogénation de cet acide dépasse en général 90\% (Bauchart et al., 1987). Les faibles proportions de ces 2 acides dans le contenu duodénal sont une constante des différentes expérimentations incluant l'analyse de la composition en acides gras duodénaux. Toutefois, un essai de Murphy et al. (1987) avec des graines de colza avait mis en évidence un total pouvant atteindre près de $10 \%$ de ces 2 acides gras dans le mélange d'acides gras à 18 atomes de carbone; la présentation sous forme de graine avait pu occasionner une protection partielle contre l'hydrogénation.
Le pourcentage élevé d'acide oléique à toutes les heures de la journée pour les 2 vaches constitue un résultat surprenant, en particulier pour le régime $H$. Jusqu'à présent, dans les essais menés avec des régimes conventionnels ou supplémentés en sources lipidiques réputées non protégées, seuls Outen et al. (1975) avec de l'herbe et Bauchart et al. (résultats non publiés) avec des régimes supplémentés en huile de colza ont signalé une proportion d'acide oléique atteignant $30 \%$ dans le mélange d'acides gras à 18 atomes de carbone. Une explication pourrait, pour le régime $H$, être une modification du métabolisme des lipides dans le rumen liée à un afflux trop important ou trop rapide de lipides, plus particulièrement d'acides gras polyinsaturés.

Le rapport stéarique/oléique, qui augmente dès la distribution d'ensilage de maïs, ne varie que faiblement par la suite, même après l'introduction ruminale des sources lipidiques. Ce résultat surprenant est en désaccord avec les observations de Steele (1983), qui montraient une augmentation très importante de la proportion d'acide oléique après le repas. Par ailleurs, on remarque les évolutions parallèles des proportions d'acide propionique dans les AGV du rumen et d'acide oléique dans les acides gras duodénaux à 18 atomes de carbone.

\section{CONCLUSION}

Les conditions de ce premier essai, réalisé sur un nombre réduit d'animaux, ne permettent pas de caractériser les sources lipidiques utilisées. II est toutefois possible de définir quelques éléments de la mise au point d'une méthode d'évaluation de l'hydrogénation des lipides. En premier lieu, la 
technique utilisée pourrait être appliquée en effectuant un nombre limité de prélèvements au cours de la journée, et cela indépendamment de l'heure de distribution des repas. Une vérification de l'évolution journalière du rapport stéarique/oléique est cependant nécessaire sur un nombre plus élevé de données. En second lieu, la technique d'apport massif de lipides dans le rumen pourrait entraîner les variations du métabolisme ruminal des acides gras par rapport à une ingestion plus modérée et plus progressive, ce qui est le cas général. Enfin, si l'on désire comparer l'action sur la digestion ruminale de différentes sources de matières grasses, il semble indispensable de les caractériser non seulement par la mesure de l'hydrogénation, ce qui est souvent préconisé, mais aussi par les orientations des fermentations des glucides.

\section{RÉFÉRENCES}

Bauchart D., Doreau M. \& Kindler A. (1987) Effects of fat and lactose supplementations on digestion in dairy cows. II longchain fatty acids. $J$. Dairy Sci. 70, 71-80

Bauchart D., Doreau M. \& Legay-Carmier F. (1985) Utilisation digestive des lipides et conséquences de leur introduction sur la digestion du ruminant. Bull. Tech. CRVZ Theix, INRA 61, 6577

Bauchart D., Legay-Carmier F., Doreau M. \& Gaillard B. (1989) Lipid metabolism of liquid- associated and solid-adherent bacteria in rumen contents of dairy cows offered lipidsupplemented diets. Br. J. Nutr. (sous presse)

Brumby P.E. \& Storry J.E. (1976) Production and testing of protected lipid supplements. Rep. Natt. Inst. Res. Dairying, 75

Doreau M., Voisin A., Elmeddah Y. \& Bauchart D. (1989) La "protection" des matières grasses utilisées dans l'alimentation des ruminants : conséquences sur la digestion. Rev. Fr. Corps Gras (sous presse)

Folch J., Lees M. \& Sloane S.M. (1957) A simple method for the isolation and purification of total lipides from animal tissues. J. Biol. Chem. 226, 497-509

Jenkins T.C. \& Palmquist D.L. (1984) Effect of fatty acids on calcium soaps on rumen and total nutrient digestibility of dairy rations. J. Dairy Sci. 67, 978-986

Jouany J.P. (1982) Volatile fatty acid and alcohol determination in digestive contents, silage juices, bacterial cultures and anaerobic fermentor contents. Sci. Alim. 2, 131-144

Legay-Carmier F. \& Bauchart D. (1989) Distribution of bacteria in rumen contents of dairy cows given a diet supplemented with soya-bean oil. Br. J. Nutr. 61 (sous presse)

Murphy J.J., Uden P., Palmquist D.L. \& Wiktorsson $\mathrm{H}$. (1987) Rumen and total diet digestibilities in lactating cows fed diets containing full-fat rapeseed. J. Dairy Sci. 70, 1572-1582

Outen G.F., Beever D.F., Osbourn D.F. \& Thomson D.J. (1975) The digestion of the lipids of processed red clover herbage by sheep. $J$. Sci. Food Agric. 26, 1381-1389

Steele W. (1983) Intestinal absorption of fatty acids and blood lipid composition in sheep. $J$. Dairy Sci. 66, 520-527 